\title{
Competition between Polysaccharide/Polynucleotide Complexation vs. Polynucleotide Hybridization; Salt Concentration Dependence of the Reaction Direction*
}

\author{
Ryouji Karinaga, Kazuya Koumoto, Jusaku Minari, Masami Mizu, Takahisa Anada, and Kazuo Sakurai ${ }^{\dagger}$ \\ Department of Chemical Process 8 Environments, The University of Kitakyushu, \\ 1-1, Hibikino, Wakamatu-ku, Kitakyushu, Fukuoka 808-0135, Japan \\ Seiji Shinkai \\ Faculty of Engineering, Department of Chemistry \& Biochemistry, \\ Graduate School of Engineering, Kyushu University, \\ 6-10-1 Hakozaki, Higashi-ku, Fukuoka, Fukuoka 812-8581, Japan \\ (Received 23 December 2004; Accepted 18 January 2005; Published 7 February 2005)
}

\begin{abstract}
Schizophyllan is a natural $\beta$ - $(1 \rightarrow 3)$-D-glucan existing as a triple helix in water and as a single chain in dimethylsulfoxide (DMSO), respectively. As we already reported, when a homo-polynucleotide [ex. poly (A) or poly $(\mathrm{dA})$ ] was added to the schizophyllan/DMSO solution and subsequently DMSO was exchanged for water, the single chain of schizophyllan formed a complex with the polynucleotide. In this paper, using an adenine-thymine (or uracil) double strand as a model system, we explored whether s-SPG can unzip the polynucleotide duplexes, examining the competition between the schizophyllan/polynucleotide complexation and the polynucleotide hybridization with circular dichroism and fluorescence spectroscopy at various $\mathrm{NaCl}$ concentrations. The unzipping reaction happened for the hetero-duplexes (i.e., RNA-DNA duplexes) at low salt concentrations, on the other hand, it was not observed for the homo-duplexes at all the salt concentrations. When we compared dissociation temperatures, the unzipping reaction of the homo-duplexes was expected at low salt concentrations. This discrepancy between the experimental results and the expectation, as well as the different behavior between the hetero- and homo- duplexes, can be ascribed to the difference in the activation energy of intermediate states. [DOI: 10.1380/ejssnt.2005.38]
\end{abstract}

Keywords: Biological molecules - nucleic acids; Biological aspects of nano-structures; Biological compounds; Hydrides; Compound formation

\section{INTRODUCTION}

Breakage (or unzipping) of base pairs of DNA is the first step of DNA replication process [1]. The unzipping reaction is basically replacement of the Watson-Crick hydrogen bonding pair by another hydrogen bond. In nature, a protein called helicase is involved in this process, consuming some energy generated by hydrolysis of ATP $[2,3]$. When we design an artificial (or alternative) compound that enables to unzip DNA in aqueous solutions, the compound has to equip not only hydrogen bonding sites but also molecular specificity to recognize bases. This is because, to form a specific hydrogen bond in aqueous solutions, the thermodynamic free energy has to be more favorable for the specific paring than for the water/compound hydrogen bonding pairs. The thermodynamic favorite has to be overwhelming to compete with the solvent water molecules. For such a specific interaction, we need allosteric effects, polymeric cooperative effects, and/or combination of hydrophobic effects.

Sakurai and Shinkai found that $\beta$ - $(1 \rightarrow 3)$-D-glucan schizophyllan can form a complex with polynucleotides in aqueous solutions [4-6]. Schizophyllan is an extracellular polysaccharide produced by the fungus Schizophyllan commune and the main chain consists of $\beta-(1 \rightarrow 3)$ D-glucan and one $\beta$ - $(1 \rightarrow 6)$-D-glycosyl side chain links to the main chain at every three glucose residues [see

\footnotetext{
*This paper was presented at International Symposium on Nano-organization and Function, Tokyo, Japan, 11-12 November, 2004; This is the 38th paper in the series of 'Polysaccharide/Polynucleotide Complexes' study.

†Corresponding author: sakurai@env.kitakyu-u.ac.jp
}

Fig. 1(a) for the chemical structure] [7]. Schizophyllan adopts a triple-helical conformation in water and a random coil in dimethylsulfoxide (DMSO) [8, 9]. When water is added to the DMSO solution (renaturation), the triple helix of schizophyllan (t-SPG) can be partially retrieved through this process (denaturation), although the entire chain structure is not the same as that of the original triple helix [10]. The single chain of schizophyllan (s-SPG) forms a macromolecular complex with some homo-phosphodiester polynucleotides [such as poly $(\mathrm{C})$, $\operatorname{poly}(\mathrm{A}), \operatorname{poly}(\mathrm{U}), \operatorname{poly}(\mathrm{dA})$, and $\operatorname{poly}(\mathrm{dT})]$, when the polynucleotide is present in the renaturation process. The renaturation, denaturation, and complexation processes and their relationship are schematically illustrated in Fig. 1(b) $[4-6]$.

Recently we found that other $\beta$ - $(1 \rightarrow 3)$-D-glucans, such as curdlan and lentinan, can also form the same kind of complexes as schizophyllan; therefore, this complexation can be regarded as a general nature for $\beta-(1 \rightarrow 3)$-Dglucans [11]. Some of the novel features for this complex are (1) the complex is remarkably stable (large binding constant) and considerably water-soluble in the physiological conditions, (2) the complexation occurs in a highly stoichiometrical manner and the stoichiometric number indicates that two schizophyllan units and three base units are interacting with each other [5]. In another word, two main chain glucoses and one base are bound by three hydrogen bonds [see Fig. 1(c)] [12, 13], and (3) the complex is automatically dissociated when $\mathrm{pH}$ becomes less than 6.0 , because protonation of the nucleotide base induces conformational changes, which cause dissociation of the complex [12].

The preceding paper [14] revealed that when a complex meets the corresponding complementary sequence in physiological solutions, for example 2 s-SPG/poly $(\mathrm{dA})$ 
(a)

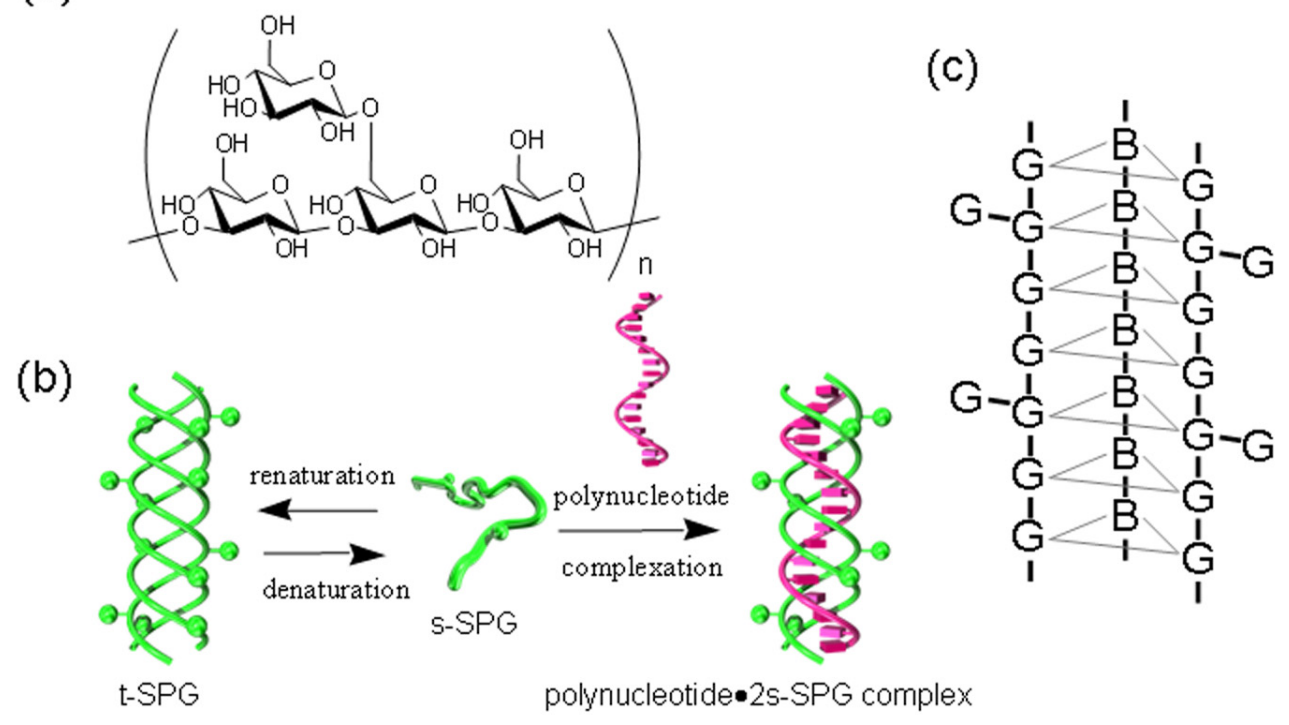

FIG. 1: (a) Repeating unit of Schizophyllan (b) Schematic illustration of the complex formation between a polynucleotide and schizophyllan. (c) Stoichiometric model for the polynucleotide/schizophyllan complex. G and B indicate the main-chain glucoses of schizophyllan and the base molecules of polynucleotide, respectively.

meets poly $(\mathrm{dT})$, the complex is dissociated immediately and hybridization takes place. This property is considered to be a great advantage when the complex is applied to antisense carriers because the bound oligonucleotides can be released when the complex encounters the target mRNA [15, 16]. Another remaining issue is whether sSPG can unzip polynucleotide duplexes, and this paper examines this concerned problem by using the adeninethymine (or uracil) base pair as a model system [17].

\section{EXPERIMENTAL}

The sample of t-SPG $\left(M_{w}=4.5 \times 10^{5}\right.$, where $M_{w}$ is the weight-average molecular weight) was kindly supplied from Taito Co. in Japan. Poly(dA), poly(A), poly $(\mathrm{dT})$, and poly $(\mathrm{U})$ were purchased from Amersham Japan, and the base number for these samples were 310 , 320,359 , and 320 , respectively, according to the sedimentation velocity. $\mathrm{dT}_{40}$ and 5'-end FITC attached $\mathrm{dA}_{40}$ (denoted by $\mathrm{dA}_{40} \mathrm{~F}$ ) were synthesized at Hokkaido System Science (Hokkaido, Japan) and purified with highpressure liquid chromatography. Sterile water and a spectroscopic grade DMSO were obtained from Kishida Chemicals and used for all measurements. 1M Tris buffer (pH 8.0, DNase and RNase free) was purchased from Wako Pure Chemical Industries, Ltd. Ethidium bromide (EtBr) was purchased from Wako Pure Chemical Industries, Ltd. The samples were prepared according to the reported method. Unless stated, the sample concentrations were as follows; $[\mathrm{poly}(\mathrm{X})]=3.7 \times 10^{-5} \mathrm{~mol} \cdot \mathrm{dm}^{-3} /$ unit and $[\mathrm{s}-\mathrm{SPG}]=2.5 \times 10^{-5} \mathrm{~mol} \cdot \mathrm{dm}^{-3} /$ repeating unit, where poly $(\mathrm{X})$ means the polynucleotide samples. After mixed s-SPG and poly $(\mathrm{X})$, the concentration ratio was controlled equal to the stoichiometric number. The volume fraction of water in the water-DMSO mixture was controlled to be 0.90 for all measurements. The circular dichroism
(CD) in 240-340 $\mathrm{nm}$ region was measured on a Jasco J820 spectropolarimeter with a $1 \mathrm{~cm}$ cell equipped with a water-jacket. From the temperature dependence of CD data upon heating, we determined the dissociation temperature $\left(T_{m}\right)$ for the duplexes and complexes. Fluorescence spectroscopy and degree of fluorescence depolarization were carried out with a Hitachi F-4500 fluorescence spectrometer and a JASCO FP-715, respectively.

\section{RESULTS AND DISCUSSION}

\section{A. Changes in CD Spectra upon Mixing with s-SPG; Proof for Unzipping of Duplexes}

Figure 2 (a-1-4) compares CD spectra between before and after addition of s-SPG into four types of nucleotide duplexes (dA-dT, rA-rT, dA-rU, and dT-rA) in a nonsalt $10 \mathrm{mM}$ Tris buffer. The homo-duplexes (dA-dT and rA-rT) show no spectral change at all upon mixing with sSPG; on the other hand, the hetero-duplexes (dA-rU and dT-rA) do show changes. Especially, dA-rU shows dramatical changes. As shown in the panel (b), the spectrum of dA-rU after addition of s-SPG can be deconvoluted into two spectra of dA•2s-SPG and $\mathrm{rU}$, indicating that the following reaction takes place automatically and the reaction direction dominantly inclined to the right side.

$$
\mathrm{dA}-\mathrm{rU}+2 \mathrm{~s}-\mathrm{SPG} \longrightarrow \mathrm{dA} \bullet 2 \mathrm{~s}-\mathrm{SPG}+\mathrm{rU} \text {. }
$$

This equation means that s-SPG can unzip a polynucleotide duplex and form an 2s-SPG/polynucleotide complex. $T_{m}$ values of $\mathrm{dA}-\mathrm{rU}$ and $\mathrm{dA} \bullet 2 \mathrm{~s}-\mathrm{SPG}$ in this solution were determined to be 35 and $65^{\circ} \mathrm{C}$, respectively. Therefore, the thermodynamic stability of $\mathrm{dA}-\mathrm{rU}$ is less than that of $\mathrm{dA} \bullet 2 \mathrm{~s}-\mathrm{SPG}$, thus direction of the reaction (1) is consistent with the difference in $T_{m}$ between the 
(a)

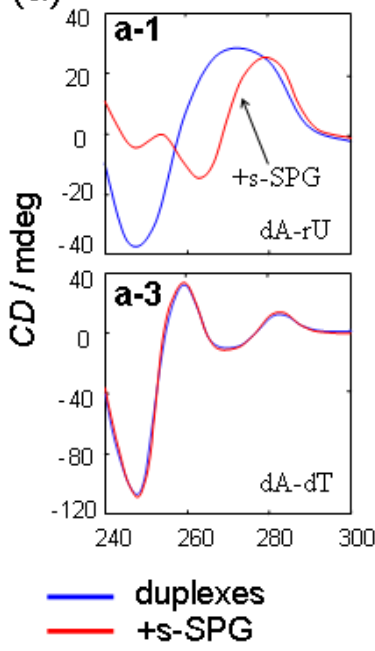

(b) (c)

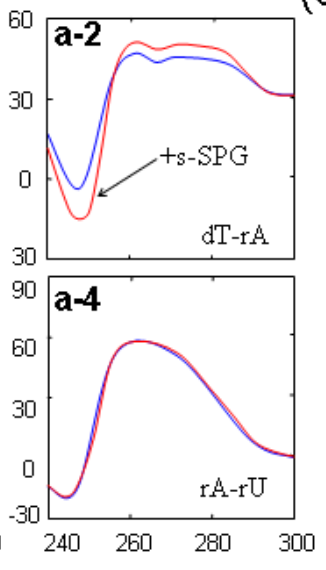

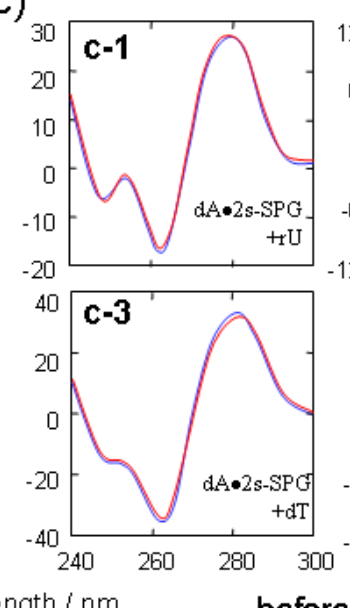
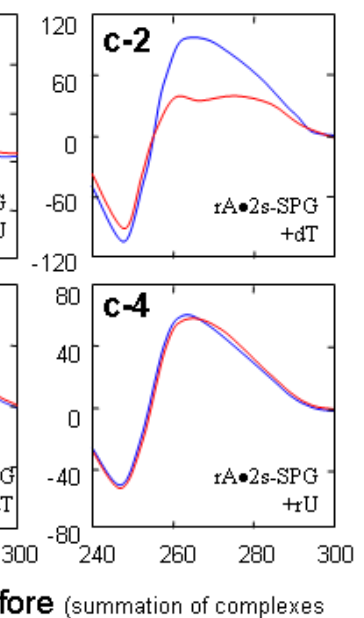

and polynucleotides)

after (measure ment)

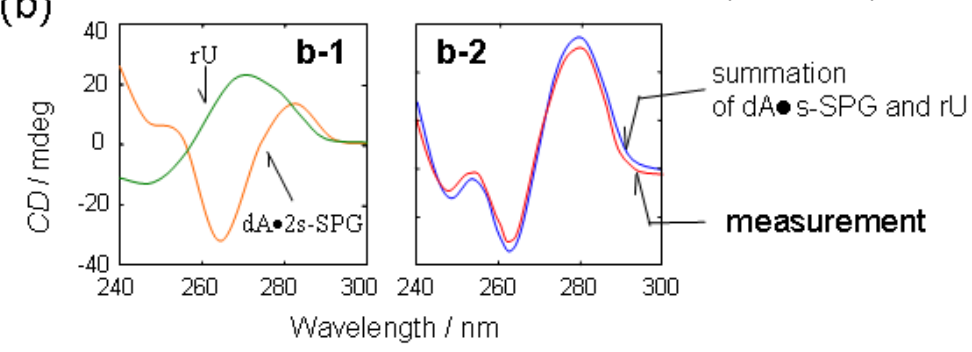

FIG. 2: Changes of CD spectra when s-SPG was added to dA-rU (a-1), dT-rA (a-2), dA-dT (a-3), or rA-rU (a-4) in non-salt $10 \mathrm{mM}$ Tris solutions, and when $\mathrm{rU}$ was added to dA•2s-SPG (c-1), dT was added to rA•2s-SPG (c-2), dT was added to $\mathrm{dA} \bullet 2 \mathrm{~s}-\mathrm{SPG}(\mathrm{c}-3)$, or $\mathrm{rU}$ was added to $\mathrm{rA} \bullet 2 \mathrm{~s}-\mathrm{SPG}(\mathrm{c}-4)$ in non-salt $10 \mathrm{mM}$ Tris solutions. The individual CD spectra for $\mathrm{rU}$ and $\mathrm{dA} \bullet 2 \mathrm{~s}-\mathrm{SPG}$ are presented in (b-1), and (b-2) compares the summation of them and the measured one when we added s-SPG to dA-rU. Their spectra were measured at $5^{\circ} \mathrm{C}$ after there samples were incubated overnight.

two spices. As presented in the panel (a-2), the CD spectral changes of dT-rA upon mixing with s-SPG seem less obvious than that of dA-rU. Deconvolution with dT•2s$\mathrm{SPG} \& \mathrm{rA}$ or $\mathrm{rA} \bullet 2 \mathrm{~s}-\mathrm{SPG} \& \mathrm{dT}$ could not fit the data. In fact, the spectrum can be deconvoluted with about $90 \%$ of dT-rA and $10 \%$ of $\mathrm{rA} \bullet 2 \mathrm{~s}-\mathrm{SPG}$ and $\mathrm{dT}$, apparently containing dT-rA as a major component, and additionally $\mathrm{rA} \bullet 2 \mathrm{~s}-\mathrm{SPG}$ and $\mathrm{dT}$. Therefore, we can express this change with the following equation, showing the reaction is inclined to the left side or in equilibrium.

$$
\mathrm{dT}-\mathrm{rA}+2 \mathrm{~s}-\mathrm{SPG} \rightleftarrows \mathrm{rA} \bullet 2 \mathrm{~s}-\mathrm{SPG}+\mathrm{dT} .
$$

$T_{m}$ values of dT-rA and rA•2s-SPG in $10 \mathrm{mM}$ Tris were determined to be 48 and $35^{\circ} \mathrm{C}$, respectively; again, the reaction direction is consistent with the magnitude order of $T_{m}$. Using the same style, unchanged CD spectra of the panels (a-3) \& (a-4) for the two homo-duplexes (dA-dT and rA-rT) can be expressed by

$$
\begin{aligned}
& \mathrm{dT}-\mathrm{dA}+2 \mathrm{~s}-\mathrm{SPG} \stackrel{\times}{\longrightarrow} \mathrm{dA} \bullet 2 \mathrm{~s}-\mathrm{SPG}+\mathrm{dT}, \\
& \mathrm{rU}-\mathrm{rA}+2 \mathrm{~s}-\mathrm{SPG} \stackrel{\times}{\longrightarrow} \mathrm{rA} \bullet 2 \mathrm{~s}-\mathrm{SPG}+\mathrm{rU} .
\end{aligned}
$$

Here, since we have already known that rU does not form a complex with s-SPG in non-salt solutions, we eliminate the possibility of $\mathrm{rU} \bullet 2 \mathrm{~s}-\mathrm{SPG}$ formation in the equations. Eqs. (3) and (4) do not necessarily mean that the reverse reactions (such as $\mathrm{rA} \bullet 2 \mathrm{~s}-\mathrm{SPG}+\mathrm{rU} \longrightarrow \mathrm{rU}-\mathrm{rA}+$
2s-SPG) are inhibited, because the experiments of panels (a-3) and (a-4) do not provide any information about the reverse reactions.

The panels (c-1-4) in Fig. 2 present how the CD spectra change when s-SPG/polynucleotide complexes are mixed with the corresponding complementary polynucleotide. As presented in the panel (c-1), the spectrum was not changed at all, when we added $\mathrm{rU}$ to dA•2s-SPG. This means that the amount of dA•2s-SPG was not changed when added $\mathrm{rU}$, confirming that the Eq. (1) is valid. The panel (c-2), i.e., rA $\bullet 2 \mathrm{~s}-\mathrm{SPG}+\mathrm{dT}$, shows some change and, again, the resultant spectrum can be deconvoluted into $\mathrm{dT}-\mathrm{rA}, \mathrm{rA} \bullet 2 \mathrm{~s}-\mathrm{SPG}$, and dT spectra, being consistent with

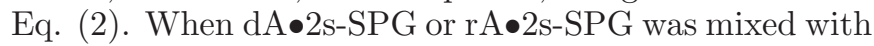
$\mathrm{dT}$ or $\mathrm{rU}$, respectively, nothing happened (panels c-3 and 4 ), thus we can describe this system with the following equations by taking the results of Eqs. (3) and (4) into account.

$$
\begin{aligned}
\mathrm{dT}-\mathrm{dA}+2 \mathrm{~s}-\mathrm{SPG} & \rightleftarrows{ }_{\times}^{\times} \rightleftarrows \mathrm{dA} \bullet 2 \mathrm{~s}-\mathrm{SPG}+\mathrm{dT}, \\
\mathrm{rU}-\mathrm{rA}+2 \mathrm{~s}-\mathrm{SPG} & \rightleftarrows{ }_{x}^{\times} \rightleftarrows \mathrm{rA} \bullet 2 \mathrm{~s}-\mathrm{SPG}+\mathrm{rU} .
\end{aligned}
$$

\section{B. Salt Concentration Dependence of Unzipping Duplexes}

Electrostatic repulsion between the anions of polynucleotide duplexes un-stabilizes the duplexes and lowers 
TABLE I: Salt concentration dependent of zipping/unzipping at $5^{\circ} \mathrm{C}$.

$$
\begin{array}{lll}
\mathrm{X}-\mathrm{Y}+\mathrm{s}-\mathrm{SPG} \longrightarrow & \mathrm{X} \bullet 2 \mathrm{~s}-\mathrm{SPG}+\mathrm{Y} & \text { Predominantly right direction }(\mathrm{PR}) \\
\mathrm{X}-\mathrm{Y}+\mathrm{s}-\mathrm{SPG} \leftarrow \longrightarrow & \mathrm{X} \bullet 2 \mathrm{~s}-\mathrm{SPG}+\mathrm{Y} & \text { Equilibrium }(\mathrm{E}) \\
\mathrm{X}-\mathrm{Y}+\mathrm{s}-\mathrm{SPG} & \mathrm{X} \bullet 2 \mathrm{~s}-\mathrm{SPG}+\mathrm{Y} & \text { Predominantly left direction }(\mathrm{PL}) \\
\mathrm{X}-\mathrm{Y}+\mathrm{s}-\mathrm{SPG} \leftarrow \mathrm{X} & \mathrm{X} \bullet 2 \mathrm{~s}-\mathrm{SPG}+\mathrm{Y} & \text { Nothing happen }(\mathrm{NH})
\end{array}
$$

\begin{tabular}{ccccc}
\hline \hline NaCl Concentration $(\mathrm{mM})$ & $\mathrm{dA}-\mathrm{dT}$ & $\mathrm{rA}-\mathrm{rU}$ & $\mathrm{dT}-\mathrm{rA}$ & $\mathrm{E}$ \\
\hline 0 & $\mathrm{NH}$ & $\mathrm{NH}$ & $\mathrm{E}$ & $\mathrm{PR}$ \\
1 & $\mathrm{E}$ & $\mathrm{NH}$ & $\mathrm{E}$ & $\mathrm{PR}$ \\
10 & $\mathrm{E}$ & $\mathrm{E}$ & $\mathrm{PL}$ & $\mathrm{E}$ \\
25 & $\mathrm{PL}$ & $\mathrm{PL}$ & $\mathrm{PL}$ & $\mathrm{E}$ \\
100 & $\mathrm{PL}$ & $\mathrm{PL}$ & $\mathrm{PL}$ & $\mathrm{E}$ \\
800 & $\mathrm{PL}$ & $\mathrm{PL}$ & $\mathrm{PL}$ & $\mathrm{PL}$ \\
1000 & $\mathrm{PL}$ & $\mathrm{PL}$ & $\mathrm{PL}$ & \\
\hline \hline
\end{tabular}

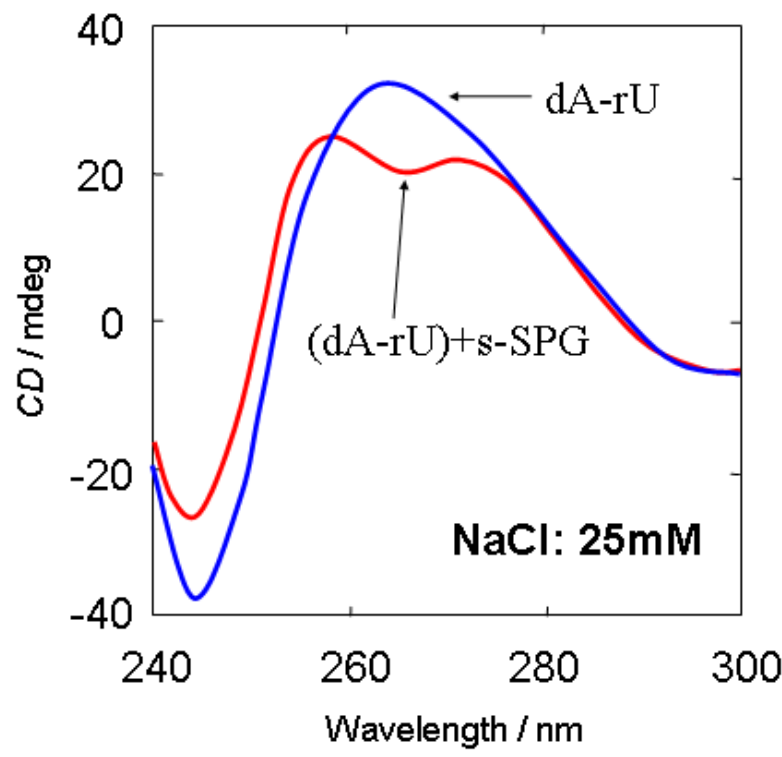

FIG. 3: Comparison of CD spectra between before and after addition of s-SPG to dA-rU at $5^{\circ} \mathrm{C}$ in a $25 \mathrm{mM} \mathrm{NaCl}$ solution.

$T_{m}$. When ionized salts are present in the solution, they can shield the repulsion (electrostatic shielding effect), thus the duplex stability is increased; in another word, $T_{m}$ is increased with increasing the salt concentration. On the other hand, at considerably low and non-salt solutions, the duplexes are not able to maintain the helix. For example, we found that $5 \mathrm{mM} \mathrm{NaCl}$ was a critical ionic strength for the dA-dT duplex. At $5 \mathrm{mM} \mathrm{NaCl}, T_{m}$ was $50^{\circ} \mathrm{C}$ and it increased to $90^{\circ} \mathrm{C}$ at $1 \mathrm{M} \mathrm{NaCl}$. Since sSPG has no charge, the polynucleotide/s-SPG complexes are stable even at non-salt conditions and the salt concentration dependence of $T_{m}$ is less sensitive than that of polynucleotide duplexes (see Fig. 4(b)) [18].

For dA-rU duplexes in a $25 \mathrm{mM} \mathrm{NaCl}$ solution, we carried out the same type of CD measurements as Fig. 2 and the results are plotted in Fig. 3. There is a large difference in the spectrum between the non-salt and $25 \mathrm{mM}$ $\mathrm{NaCl}$ solutions. In fact, the spectrum of $25 \mathrm{mM} \mathrm{NaCl}$ could be deconvoluted into three components of dA-rU, $\mathrm{dA} \bullet 2 \mathrm{~s}-\mathrm{SPG}$, and $\mathrm{rU}$ rather than into dA $\bullet 2 \mathrm{~s}-\mathrm{SPG}$ and $\mathrm{rU}$. From this deconvolution, we found that about $10 \mathrm{~mol} \%$ of dA-rU was converted to $\mathrm{dA} \bullet 2 \mathrm{~s}-\mathrm{SPG}$. We examined the salt concentration dependence for all combinations and summarize the results in Table 1.

The red region shows that the unzipping reaction can take place. It seems that the hetero-duplexes are more easy to be unzipped by s-SPG. Low salt concentrations provide advantage to s-SPG/polynucleotide complex formation, especially, the reaction lies predominantly to the right side for the dA-rU system. At $0 \mathrm{mM} \mathrm{NaCl}$ (although there is still $10 \mathrm{mM}$ Tris), polynucleotide duplexes should be very unstable. Even though, interestingly, nothing happened for the homo-duplexes. When we added extra amount of s-SPG to these duplexes, the results were not changed. With increasing the salt concentration, as expected, duplex formation becomes more favorable than that of the complexation, and at $1 \mathrm{M}$, the reactions lie predominantly to the left side.

Figure 4(a) plots the CD melting curves for dA-rU and $\mathrm{dA}-\mathrm{rU}+\mathrm{s}-\mathrm{SPG}$, comparing between $0 \mathrm{mM}$ (upper) and 25 $\mathrm{mM}$ (lower) solutions. In the $0 \mathrm{mM}$ solution, the $\mathrm{dA}$ $\mathrm{rU}$ duplex is dissociated between 30 and $40^{\circ} \mathrm{C}$. From the middle point method, $T_{m}$ can be determined to be $35^{\circ} \mathrm{C}$. In the same salt concentration, all duplexes of dA-rU+s$\mathrm{SPG}$ were converted to a mixture of $\mathrm{dA} \bullet 2 \mathrm{~s}-\mathrm{SPG}$ and $\mathrm{rU}$. Therefore, there is no abrupt change due to the dissociation of $\mathrm{dA}-\mathrm{rU}$ around $35^{\circ} \mathrm{C}$. The $\mathrm{CD}$ values dramatically decreases above $60^{\circ} \mathrm{C}$, corresponding to the dissociation of $\mathrm{dA} \bullet 2 \mathrm{~s}-\mathrm{SPG}$. When we increased the salt concentration to $25 \mathrm{mM}, T_{m}$ of dA-rU was increased to $40^{\circ} \mathrm{C}$ owing to the electrostatic shielding. The dA-rU+s-SPG solution at this salt concentration presents two CD drops corresponding to the dissociations of $\mathrm{dA}-\mathrm{rU}$ and $\mathrm{dA} \bullet 2 \mathrm{~s}-\mathrm{SPG}$. One may notice that, for the mixture of dA-rU+s-SPG at 25 $\mathrm{mM}$, the lower $T_{m}$ shifts toward the high temperature and the higher $T_{m}$ stays the same position as that of dA•2sSPG. One possible explanation for this phenomenon can be given, assuming that the chain length fractionation was occurred and the longer dA chains only form duplexes.

Figure $4(\mathrm{~b})$ plots $T_{m}$ against the $\mathrm{NaCl}$ concentration, including the data points cited from the previous work [18] for rA-rU systems. At low concentrations, $T_{m}$ values of duplexes are lower than those of dA $\bullet 2$ s-SPG complexes, and with increasing the concentration, they are increased slightly. Around 0.1-1.0 M, they cross and above these concentrations, $T_{m}$ values of duplexes are higher than those of the dA $\bullet 2$ s-SPG complex. This feature can be renationalized with the electrostatic shielding effect. 
(a)
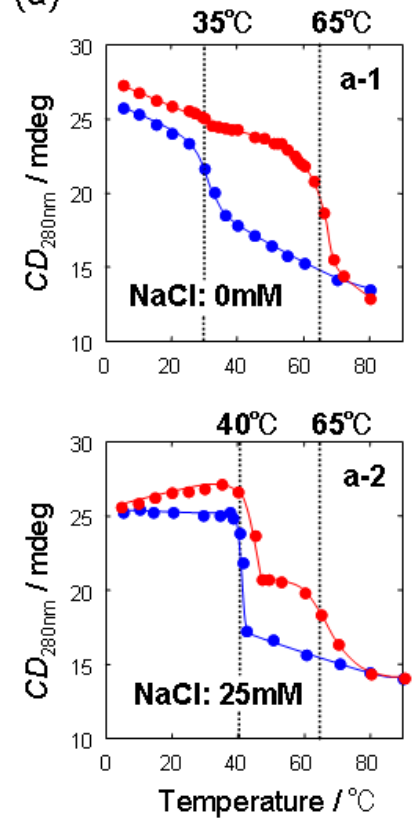

- $\mathrm{dA}-\mathrm{rU} \bullet(\mathrm{dA}-\mathrm{rU})+\mathrm{s}-\mathrm{SPG}$ (b)

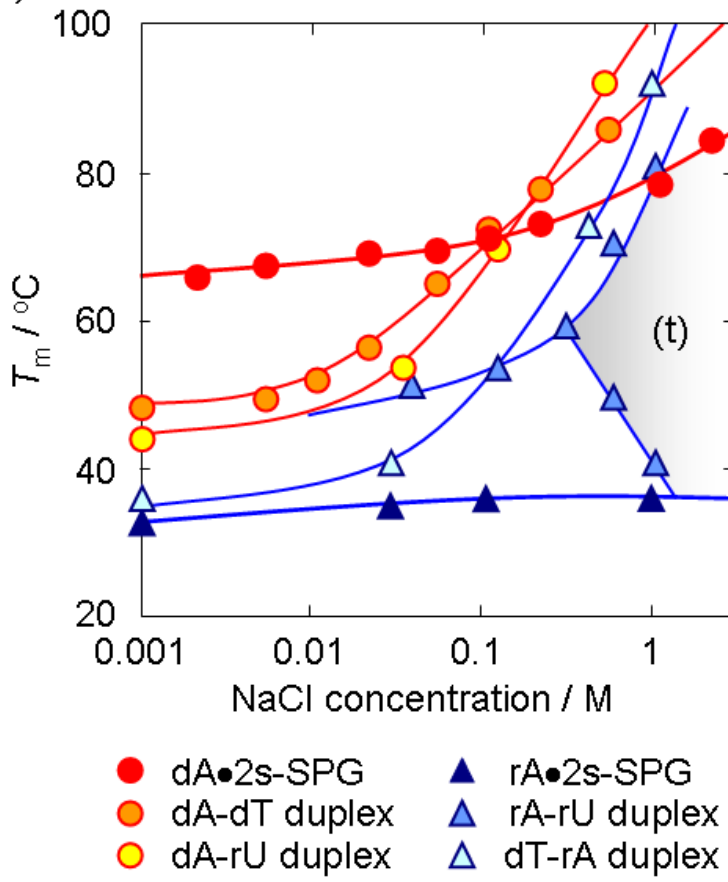

FIG. 4: Melting curves for dA-rU and dA-rU+s-SPG at the non-salt condition (a-1) and at $25 \mathrm{mM} \mathrm{NaCl}$ (a-2), and the plots of the dissociation temperature $\left(T_{m}\right)$ against the salt concentration (b). The shaded region $(\mathrm{t})$ is where poly $(\mathrm{A}) / \mathrm{poly}(\mathrm{U}) / \mathrm{poly}(\mathrm{U})$ triplex is formed.

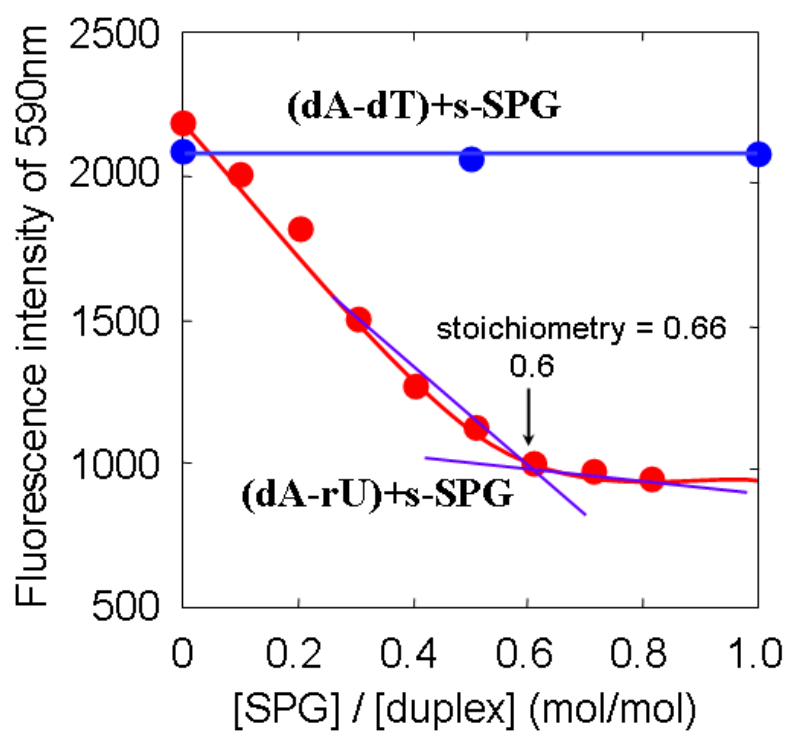

FIG. 5: Fluorescence intensity decay of the EtBr intercalated in the duplexes when the unzipping reaction of dA-rU occurs.

This effect reduces repulsion between the anions in the same nucleotide for the complex. On the other hand, it reduces both inter- and intra- molecular repulsions for the case of polynucleotide duplexes. Therefore, the shielding effect provides more impact on the duplexes than on the complexes. According to the previous work, the stability order of polynucleotide duplexes in physiological conditions are RNA duplex $>$ r(purine).d(pyrimidine) hybrid $>$ DNA duplex $>d$ (purine).r(pyrimidine) hybrid [19, 20].

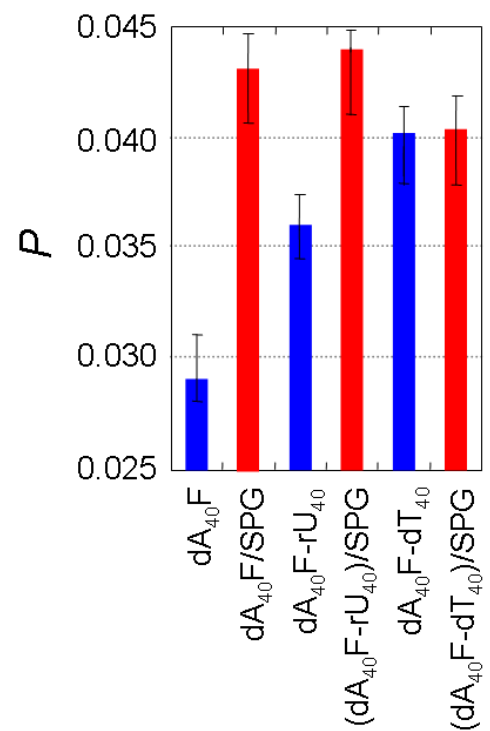

FIG. 6: Comparison of the fluorescence depolarization $(P)$.

The $\mathrm{NaCl}$ concentration of $35 \mathrm{mM}$ corresponds to physiological conditions, and the order of the stability of the duplexes follows the general rules.

The general trends in Table 1 (i.e., the duplexes are more stable than the complexes in the lower salt and the order is reversed in the higher salt) is consistent with the comparison of $T_{m}$ in Fig. 4. However, there are some inconsistencies. The comparison predicts that the unzipping reaction might occur in the more wide salt concentration range than the observation; it should have happened 


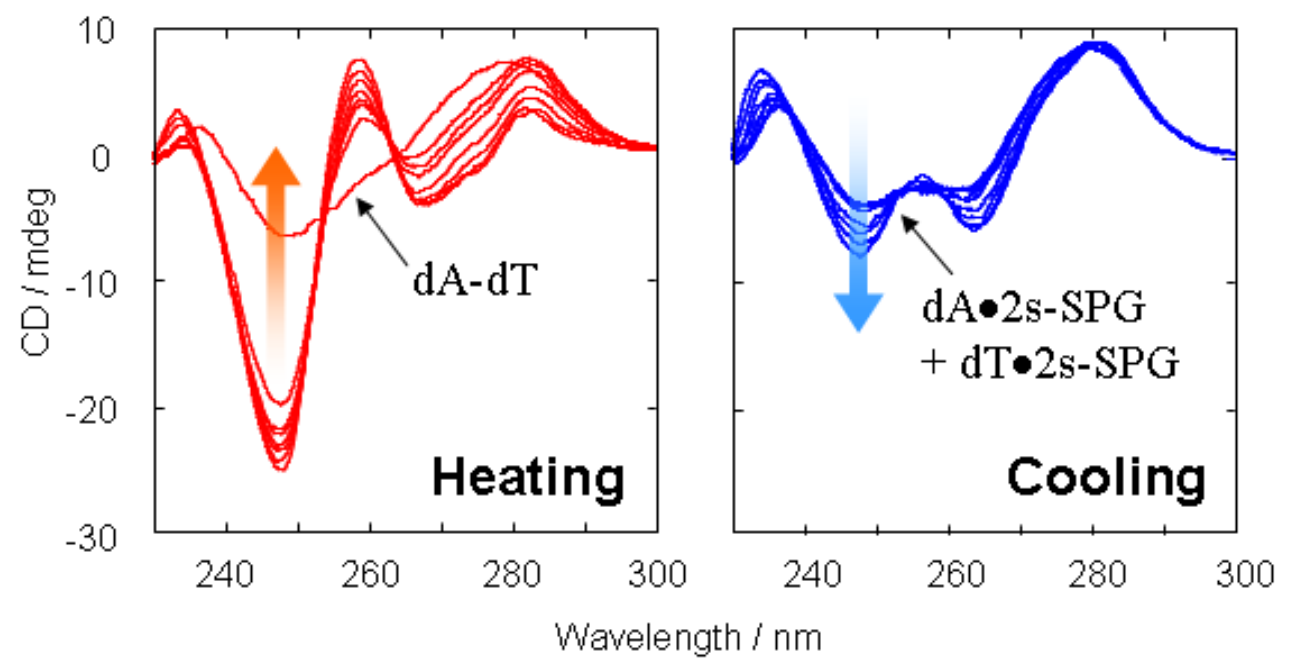

FIG. 7: Temperature dependence of the CD spectra upon heating (left) and subsequent cooling (right) for the mixture of dA-dT duplex and s-SPG. Before heating, the spectrum only consists of dA-dT and after cooled it can be deconvoluted into dA $\bullet 2$ s-SPG and dT•2s-SPG.

at less than about $100 \mathrm{mM}$. The fact is that the complete unzipping ( $\mathrm{PR}$ in the table) takes place only for $\mathrm{dA}-\mathrm{rU}$ at 0 and $1 \mathrm{mM}$. The $\mathrm{dA}-\mathrm{rU}$ shows the lowest $T_{m}$ than the other duplexes, which is qualitatively consistent with the fact that PR is only observed for this duplex. Figure 4(b) also plots $T_{m}$ for $\mathrm{rA} \bullet 2 \mathrm{~s}-\mathrm{SPG}$ complexes. The $T_{m}$ values for $\mathrm{rA} \bullet 2 \mathrm{~s}-\mathrm{SPG}$ are lower than those for all duplexes. These results are consistent with the fact that we did not observe the complete un-zipping reaction for all duplexes consisted of rA. A question is why nothing happens for the homo-duplexes at $0 \mathrm{mM}$ solution and this issue will be discussed later.

\section{Confirmation of Unzipping with Fluorescence Technique}

Ethidium bromide (EtBr) can intercalate into DNA/DNA or DNA/RNA duplexes and the intercalated EtBr gives a strong fluorescence around $590 \mathrm{~nm}$ by exciting with $260 \mathrm{~nm}$, while free EtBr gives weeker fluorescence than the intercalated one. Using this difference, the unzipping process can be detected [21]. Figure 5 plots the EtBr fluorescence intensity against the s-SPG/duplex molar ratio defined by $[\mathrm{s}-\mathrm{SPG}] /[$ duplex $]$. When we added s-SPG to dA-dT at $0 \mathrm{mM} \mathrm{NaCl}$, the fluorescence intensity does not depend on the amount of s-SPG. This feature is consistent with Eq. (5). On the other hand, when we added s-SPG to dA-rU, the intensity decreases linearly with increasing the s-SPG amount up to $[\mathrm{s}-\mathrm{SPG}] /[$ duplex $]=0.6$ and above this turnoff point the intensity becomes constant. Here, we defined [duplex] by the base-pair molar concentration and [S-SPG] by the repeating unit molar concentration, respectively. In the complex, two main chain glucoses interact with one base, and one repeating unit of s-SPG contains three main chain glucoses. Therefore, the stoichiometric amount of $\mathrm{S}-\mathrm{SPG}$ is given by $[\mathrm{s}-\mathrm{SPG}] /[$ duplex $]=0.66$. This number almost agrees with the turnoff point in Fig. 5, confirming Eq. (1).
Figure 6 compares the degree of fluorescence depolarization $(P)$. The mixture of $\mathrm{dA}_{40} \mathrm{~F}+\mathrm{s}-\mathrm{SPG}$ has a larger $P$ value than that of $\mathrm{dA}_{40} \mathrm{~F}$ itself, because $\mathrm{dA}_{40} \mathrm{~F}$ can make a complex with s-SPG, resulting in the decrease of the rotating diffusion coefficient. The $P$ value for $\mathrm{dA}_{40} \mathrm{~F} \bullet 2 \mathrm{~s}-\mathrm{SPG}$ is 0.043. Increment of $\mathrm{P}$ was also observed when we mixed sSPG with $\mathrm{dA}_{40} \mathrm{~F}-\mathrm{rU} \mathrm{U}_{40}$, and the resultant $P$ value agrees with that of $\mathrm{dA}_{40} \mathrm{~F}+\mathrm{s}-\mathrm{SPG}$, confirming that $\mathrm{dA}_{40} \mathrm{~F} \bullet 2 \mathrm{~s}-$ $\mathrm{SPG}$ is formed in the mixture. However, when we mixed with $\mathrm{dA}_{40} \mathrm{~F}-\mathrm{dT}_{40}$ and s-SPG, nothing happened, similarly to the $\mathrm{CD}$ results.

\section{Thermal Cycle and Complex vs. Duplex Formations}

The CD and fluorescence measurements showed that $\mathrm{dT}$-dA was never converted to $\mathrm{dA} \bullet 2 \mathrm{~s}-\mathrm{SPG}+\mathrm{dT}$ at 0 $\mathrm{mM} \mathrm{NaCl}$. Although, at this salt concentration, the comparison of $T_{m}$ suggested that the reaction of dT-dA $+2 \mathrm{~s}-$ $\mathrm{SPG} \longrightarrow \mathrm{dA} \bullet 2 \mathrm{~s}-\mathrm{SPG}+\mathrm{dT}$ is expected to happen. Figure 7 illustrates $\mathrm{CD}$ spectral changes when we heated dT$\mathrm{dA}+$ s-SPG (extra amount) mixture up to $80^{\circ} \mathrm{C}$ and cooled it down to room temperature. The spectrum before heating indicates that only $\mathrm{dT}-\mathrm{dA}$ is present and we found that the shape was never changed even after annealed the mixture for a week at $5^{\circ} \mathrm{C}$. When the sample was heated, the duplex dissociation was observed at $60^{\circ} \mathrm{C}$. When we heated the sample up to $80^{\circ} \mathrm{C}$, the observed spectrum can be deconvoluted into only mixture of $\mathrm{dA}$ and $\mathrm{dT}$ single chains, confirming that the duplex was dissociated. Subsequently, we cooled it down (in the presence of extra amount of s-SPG), the resultant spectrum is completely different from the original ones, as presented in the right side. The spectrum after cooled can be approximately deconvoluted into a 1:1 mixture of $\mathrm{dA} \bullet 2 \mathrm{~s}-\mathrm{SPG}+\mathrm{dT} \bullet 2 \mathrm{~s}-\mathrm{SPG}$. This result suggests that the activation energy for the reaction of $\mathrm{dT}-\mathrm{dA}+2 \mathrm{~s}-\mathrm{SPG} \longrightarrow$ $\mathrm{dA} \bullet 2 \mathrm{~s}-\mathrm{SPG}+\mathrm{dT}$ might be too large to overcome at low temperatures, despite a negative $\Delta G$ for this reaction is 


\section{Hetero-duplexes system}

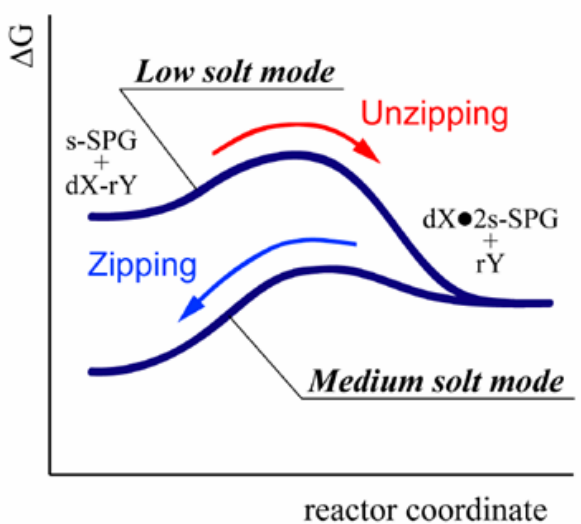

\section{Homo-duplexes system}

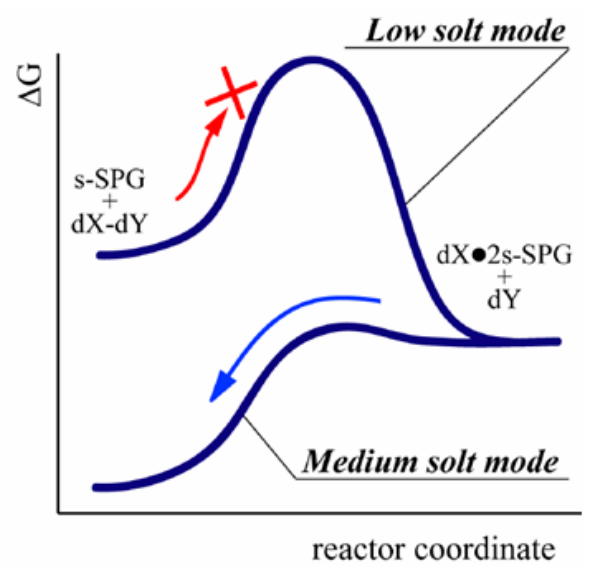

FIG. 8: Schematic illustrations of the reaction coordinates to explain difference in the experimental data (Table 1) between the homo- and hetero- duplexes.

expected.

To sum up what we observed, Fig. 8 illustrates the salt concentration dependence of the reaction directions. For the hereto-duplexes, the free energy barrier (the activation energy of intermediate states) from the duplexes to the complex (and vice versa) is easy to overcome. The free energy of the duplexes is higher than the complex at low salt concentrations, and becomes lower than the complex when the salt concentration is increased. For the homoduplexes, at the medium salt concentrations, the reaction profile is almost the same as that of the hetero-duplexes. However, the 'unzipping' reaction seems difficult to happen owing to the high energy barrier.

Assuming a simple bimolecular reaction, the transition temperature $\left(T_{m}\right)$ can be written by $T_{m}=\Delta H / \Delta S$, where $\Delta H$ and $\Delta S$ are the common notations. When we consider free energy $(\Delta G)$ for two different reactions (a and b), if $\Delta S_{a}$ and $\Delta S_{b}$ are equal, we can conclude that $T_{m, a}>T_{m, b}$ means $\Delta G_{a}<\Delta G_{b}$. However, generally, $T_{m, a}>T_{m, b}$ does not necessarily mean $\Delta G_{a}<\Delta G_{b}$. This could be a reason that quantitative agreement was not obtained between Table 1 and Fig. 4. For more detailed discussion, we may have to use the free energy which can be determined from the van't Hoff plot. Even though, the van't Hoff theory assumes a simple bimolecular reaction and equilibrium between two states. However, it may not be adequate to treat complexed systems such as the present one with such a simple model.

\section{CONCLUDING REMARKS}

We have explored whether s-SPG can unzip polynucleotide duplexes by changing the salt concentration and found that it automatically happens for hetero-duplexes at low salt concentrations. In terms of $T_{m}$, the un-zipping reaction is possible for the homo-duplexes, however, the activation energy may be too high to overcome for the unzipping reaction, suggesting that the s-SPG-induced unzipping reaction may hardly occur in nature. The present results should provide a new insight and fundamental information to design a new system such as a nucleotide separation device.

\section{Acknowledgments}

This work is finically supported by 'Organization and Function', PRESTO, and SORST programs in Japan Science and Technology Corporation (JST).
[1] T. Ogawa and T. Okazaki, Annu Rev Biochem 49, 421 (1980).

[2] R. Galletto, M. J. Jezewska and W. Bujalowski, J. Mol. Biol. 343, 83 (2004).

[3] R. Galletto, M. J. Jezewska and W. Bujalowski, J. Mol. Biol. 343, 101 (2004).

[4] K. Sakurai and S. Shinkai, J. Am. Chem. Soc. 122, 4520 (2000).

[5] K. Sakurai, M. Mizu and S. Shinkai, Biomacromolecules 2, 641 (2001).

[6] M. Mizu, T. Kimura, K. Koumoto, K. Sakurai and S. Shinkai, Chem. Commun. 2001, 429 (2001).

[7] K. Tabata, W. Ito, T. Kojima, S. Kawabata and A. Mis- aki, Carbohydr. Res. 89, 121 (1981).

[8] T. Yanaki, T. Norisuye and H. Fujita, Macromolecules 13, 1462 (1980).

[9] T. Norisuye, T. Yanaki and H. Fujita, J. Polym. Sci., Polym. Phys. Ed. 18, 547 (1980).

[10] B. T. Stokke, A. Elgsaeter, D. A. Brant, T. Kuge and S. Kitamura, Biopolymers 33, 193 (1993).

[11] T. Kimura, K. Koumoto, K. Sakurai and S. Shinkai, Chem. Lett. 31, 1242 (2002).

[12] K. Sakurai, R. Iguchi, M. Mizu, K. Koumoto and S. Shinkai, Bioorg Chem 31, 216 (2003).

[13] K. Miyoshi, K. Uezu, K. Sakurai and S. Shinkai, Chemistry \& Biodiversity 1, 916 (2004). 
[14] K. Koumoto, M. Mizu, K. Sakurai, T. Kunitake and S. Shinkai, Chemistry \& Biodiversity 1, 520 (2004).

[15] M. Mizu, K. Koumoto, T. Anada, R. Karinaga, T. Kimura, T. Nagasaki, S. Shinkai and K. Sakurai, Bulletin of the Chemical Society of Japan 77, 1101 (2004).

[16] T. Matsumoto, M. Numata, T. Anada, M. Mizu, K. Koumoto, K. Sakurai, T. Nagasaki and S. Shinkai, Biochim. Biophys. Acta 1670, 91 (2004)

[17] Notations of the Mixtures and Complexes. In this paper, we denote the hybridized duplex made of poly $(\mathrm{dA})$ and poly $(\mathrm{U})$ by dA-rU, and the complex made of poly $(\mathrm{dA})$ and s-SPG by dA $\bullet 2$ s-SPG, for example. For simplicity, we use sometimes dX or rY instead of poly $(\mathrm{dX})$ or poly $(\mathrm{rY})$, where $\mathrm{X}$ and $\mathrm{Y}$ are nucleotide base codes. The mixture made of dA-rU and s-SPG was expressed by dA-rU+ sSPG, however, it does not necessary means that the resultant solution contains dA-rU and s-SPG. The solute may be changed to mixture of dA•2s-SPG, dA-rU, and s-SPG.

[18] R. D. Blake, J. Massoulie and J. R. Fresco, J. Mol. Biol. 30, 291 (1967).

[19] S. H. Hung, Q. Yu, D. M. Gray and R. L. Ratliff, Nucleic Acids Res. 22, 4326 (1994).

[20] L. Ratmeyer, R. Vinayak, Y. Y. Zhong, G. Zon and W. D. Wilson, Biochemistry 33, 5298 (1994).

[21] N. Matsuo and P. M. Ross, Arch Biochem Biophys 266, 351 (1988). 